\title{
El papel de la historia de exposición solar y el genotipo del gen $M C 1 R$ en la acumulación de mutaciones somáticas en piel humana normal
}

\author{
Aitor Cuadros Lozano \\ al259595@uji.es \\ Bárbara Hernando Fuster \\ hernandb@uji.es \\ Conrado Martínez Cadenas \\ ccadenas@med.uji.es
}

\section{Resumen}

Introducción: La predisposición a desarrollar cáncer de piel está influenciada por factores genéticos (variantes genéticas que determinan la sensibilidad solar de la piel) y ambientales (la exposición solar). La radiación ultravioleta causa alteraciones en la secuencia del ADN genómico de las células epidérmicas. La acumulación progresiva de mutaciones somáticas contribuye al envejecimiento celular y puede llevar al cáncer. Entender cómo las mutaciones somáticas se acumulan en células normales es fundamental para conocer las etapas iniciales del desarrollo del cáncer cutáneo.

Objetivos: Analizar las mutaciones somáticas acumuladas en piel normal según su patrón de fotoexposición. Analizar si existe un riesgo añadido de acumular mutaciones somáticas en individuos portadores de variantes genéticas del gen $M C 1 R$ asociadas a una mayor sensibilidad solar.

Métodos: Se obtuvo ADN de 25 biopsias de piel normal. Mediante secuenciación dirigida, se detectaron las mutaciones somáticas acumuladas en 47 genes clave en el proceso tumoral. Se secuenció la región codificante de $M C 1 R$. Los datos fenotípicos de pigmentación y sensibilidad solar se recogieron mediante un cuestionario estandarizado. La tasa mutacional acumulada entre muestras se comparó utilizando el programa SPSS v25.

Resultados: Existe una correlación positiva entre la exposición solar acumulada y la frecuencia de mutaciones somáticas en el tejido epidérmico normal. Los individuos con variantes deletéreas del gen $M C 1 R$ no parecen acumular más mutaciones en piel normal.

Conclusiones: La historia de fotoexposición y el genotipo del gen MC1R podrían incrementar la predisposición a desarrollar cáncer de piel mediante una mayor acumulación de mutaciones somáticas en estados preneoplásicos.

Palabras clave: cáncer de piel, melanoma, exposición solar, $M C 1 R$, fototipo. 


\begin{abstract}
Introduction: The predisposition to skin cancer development is influenced by genetic (genetic variants that determine skin sensitivity to sunlight) and environmental factors (sunlight exposure). Ultraviolet radiation causes alterations in the genomic DNA of the epidermal cells. The progressive accumulation of somatic mutations contributes to cellular aging and can lead to cancer. Understanding how somatic mutations accumulate in normal cells is essential to understand the early stages of skin cancer development.

Objectives: To analyse the somatic mutations accumulated in normal skin according to the pattern of sun exposure. To analyse if there is an increased risk to accumulate somatic mutations in individuals who carry genetic variants in the $M C 1 R$ gene associated with greater skin sensitivity to sunlight.

Methods: DNA from 25 cancer-free skin biopsies was obtained. By means of targeted sequencing, the somatic mutations accumulated in 47 key genes in tumour initiation and development were detected. The coding region of $M C 1 R$ was sequenced. Phenotypic data related to pigmentation and sunlight sensitivity was collected by using a standardized questionnaire. The mutational rate accumulated of population subsets were compared using the SPSS v25 program. Results: There is a positive correlation between the level of sun exposure and the frequency of somatic mutations in normal epidermis. Individuals with deleterious variants of $M C 1 R$ do not seem to accumulate more mutations.

Conclusions: The history of sunlight exposure and the MC1R genotype may increase the predisposition to skin cancer development via an increased occurrence of somatic mutations in preneoplastic stages.
\end{abstract}

Keywords: skin cancer, melanoma, sun exposure, $M C 1 R$, phototype.

\title{
Introducción
}

Una de las enfermedades que más preocupa en la actualidad a nuestra sociedad y para la que todavía existen grandes lagunas en el conocimiento, a pesar del gran trabajo de investigación que se le está dedicando, es el cáncer. Y precisamente dentro del amplio y variado campo de la oncología, una de las neoplasias que se debe destacar es el cáncer de piel, el cual se puede clasificar en dos categorías principales: el melanoma cutáneo, que se inicia mediante la transformación maligna de los melanocitos, y el cáncer de piel no melanoma, que surge a partir de otras células epidérmicas, principalmente de los queratinocitos. Este último tipo de neoplasia puede subdividirse en otras dos clases en base a la capa epidérmica de la que se origina: el carcinoma basocelular y el carcinoma de células escamosas (Hernando 2017).

\section{Bases genéticas de la patogénesis del cáncer}

La mayoría de neoplasias emergen como consecuencia de diversas alteraciones que se producen en la secuencia de ADN genómico de las células cancerosas (Stratton, Campbell y Futreal 2009).

En primer lugar, para entender correctamente la aparición y el desarrollo del cáncer, es importante conocer qué son y en qué se diferencian una mutación en línea germinal y una mutación somática. Por un lado, una mutación en línea germinal se define como aquel cambio que ocurre en el material genético de las células productoras de 
gametos y que, como resultado, será transmitido a la siguiente generación. Por otro lado, se conoce con el nombre de mutación somática aquel cambio que se adquiere en el ADN genómico de una célula somática a lo largo de la vida del individuo. Como consecuencia, todas aquellas células que deriven de esta primera célula mutada heredarán esa alteración en su material genético. Al afectar a las células somáticas, y no a los gametos, estas mutaciones no se transmitirán a la descendencia (Karki, Pandya, Elston y Ferlini 2015; Stratton, Campbell y Futreal 2009).

A pesar de que la mayoría de las mutaciones somáticas que se acumulan en nuestras células no conllevan ningún tipo de consecuencia biológica, en ocasiones una alteración somática puede afectar a un gen o a un elemento de regulación causando trastornos en funciones celulares clave. Así pues, las mutaciones somáticas se pueden clasificar, independientemente de su naturaleza estructural, en función de su implicación en el desarrollo del cáncer y su contribución al envejecimiento celular. Se distinguen dos tipos: mutación conductora y mutación pasajera. Las mutaciones conductoras confieren una ventaja proliferativa en las células portadoras y se seleccionan positivamente en la evolución del cáncer, otorgando así una ventaja selectiva para el crecimiento del tumor. Normalmente, las mutaciones conductoras son aquellas que afectan a un subconjunto de genes clave en el desarrollo tumoral (oncogenes o genes supresores tumorales). La acumulación progresiva de mutaciones conductoras a lo largo de la vida puede llevar al cáncer y contribuir al envejecimiento celular. En cambio, las mutaciones pasajeras no confieren una ventaja selectiva a la célula $y$, por lo tanto, no causan alteraciones importantes en la función celular (Stratton, Campbell y Futreal 2009).

El ADN de las células de nuestro organismo puede verse alterado continuamente, ya sea debido a la exposición a mutágenos de origen externo o a errores endógenos que pueden ocurrir en el proceso de replicación del ADN o en la división celular. Estas mutaciones comienzan a surgir en estados preneoplásicos, en los que la célula afectada todavía no muestra características fenotípicas de una célula cancerosa. Afortunadamente, disponemos de diversos mecanismos de reparación que consiguen que la mayor parte de las alteraciones en el genoma sean reparadas. Sin embargo, la eficiencia de reparación no es total y en algunos casos estos errores pueden escapar a su reparación, logrando permanecer y acumularse (Stratton, Campbell y Futreal 2009).

Como se ha comentado anteriormente, la adquisición de una mutación puntual en el genoma no significa que el individuo vaya a desarrollar una neoplasia, sino que esta solo se podrá dar tras la acumulación de diversas mutaciones conductoras que afecten a genes que codifiquen proteínas clave en la correcta regulación del ciclo celular. Por ejemplo, mutaciones en el gen $P 53$ provocan una resistencia a la apoptosis que se traduce en la expansión clonal de los queratinocitos precancerosos. De hecho, en torno al $90 \%$ de los carcinomas de células escamosas y el $50 \%$ de los carcinomas basocelulares tienen una mutación que afectan a la función de P53 (Madan, Lear y Szeimies 2010). Del mismo modo, mutaciones en genes que codifican proteínas encargadas de corregir daños en el ADN también contribuirán a la aparición de cáncer.

\section{Papel de la radiación ultravioleta (UV) en el desarrollo del cáncer de piel}

Existen diversos factores genéticos, fenotípicos y ambientales que contribuyen a la predisposición del cáncer de piel, siendo la radiación UV uno de los principales factores implicados en su desarrollo (Scherer y Kumar 2010). La radiación UV es un agente mutagénico que tiene la capacidad de dañar la secuencia de ADN mediante la formación de enlaces covalentes entre dos pirimidinas adyacentes en una misma 
hebra de ADN, principalmente de tipo timina-timina. Este nuevo dímero de pirimidinas se conoce con el nombre de ciclobutano. La mayor parte de los dímeros se reparan de inmediato, pero algunos escapan a la reparación, pudiendo afectar a los procesos de replicación y transcripción (González-Púmariega, Vernhes Tamayo y Sánchez-Lamar 2009). Así pues, la exposición solar se relaciona con la aparición de mutaciones somáticas en las células epidérmicas que más tarde pueden dar lugar a neoplasias cutáneas.

\section{La importancia del gen MC1R en la sensibilidad cutánea a la exposición solar}

Otro factor fundamental que predispone a la aparición de cáncer de piel es la posesión de ciertas variantes genéticas en genes que afectan a la pigmentación y a la sensibilidad solar. Se han descrito más de 100 genes asociados con la pigmentación humana, siendo el gen del receptor de melanocortina $1(M C 1 R)$ el más importante en determinar el riesgo a cáncer de piel. Determinadas variantes de $M C 1 R$, denominadas variantes $\mathrm{R}$, se han relacionado con el pelo rojo, con tener una mayor sensibilidad al sol o con la presencia de pecas. Todos ellos son fenotipos de riesgo de desarrollar un cáncer de piel (Robles-Espinoza et al. 2016; Tagliabue et al. 2015). Sin embargo, la importancia de este gen no solo radica en sus efectos a nivel de la pigmentación, sino que también juega un papel fundamental en la activación de vías de reparación del ADN de los melanocitos cuando este resulta dañado por la radiación UV (GarcíaBorrón, Abdel-Malek y Jiménez-Cervantes 2014).

El receptor de melanocortina $1(M C 1 R)$ es un receptor acoplado a proteína $\mathrm{G}$ ubicado en la superficie de los melanocitos. Cuando $M C 1 R$ se activa por la unión a $\alpha$ melanocortina ( $\alpha-\mathrm{MSH})$, varias cascadas de señalización son activadas, entre ellas la vía de AMPc (adenosín monofosfato cíclico), conduciendo a la síntesis de eumelanina, así como a la reparación del daño del ADN inducido por la radiación UV y al control de la proliferación celular y la apoptosis (García-Borrón, Abdel-Malek y JiménezCervantes 2014). Las variantes $R$ afectan a la función del receptor limitando la síntesis de eumelanina. Como consecuencia, existe una mayor ratio de feomelanina (tipo de melanina de color rojo/anaranjado asociada con el pelo rojo, la piel clara, la incapacidad de broncearse y las pecas) respecto a eumelanina (melanina de color marrón oscuro o negro). Generalmente, las personas portadoras de variantes $\mathrm{R}$ en $M C 1 R$ son altamente fotosensibles y propensas a las quemaduras solares cuando se exponen a la radiación UV (Robles-Espinoza et al. 2016).

\section{Justificación del trabajo}

Este estudio pretende esclarecer cuáles son los mecanismos genéticos y moleculares que conducen a las células normales a evolucionar a células tumorales, tratando de determinar qué papel juega la aparición y la acumulación de mutaciones somáticas en genes clave en el proceso tumoral. La secuenciación de muestras neoplásicas ofrece una visión parcial del proceso tumoral, ya que deja vacíos fundamentales en la comprensión de las etapas más tempranas de desarrollo del cáncer. En la bibliografía actual apenas disponemos de información acerca de las mutaciones que podemos encontrar en tejido cutáneo sano o incluso en lesiones benignas precursoras, es decir, estadios previos al desarrollo de la lesión tumoral.

De este modo, nuestro estudio pretende analizar las diferencias en la cantidad de mutaciones entre los diferentes tejidos según la región de la que han sido extraídos y, por tanto, según el patrón de exposición solar al que se han visto sometidos. Además, 
este estudio analiza las diferencias en la acumulación de mutaciones somáticas según las variantes genéticas del gen $M C 1 R$ del individuo.

\section{Métodos}

Población del estudio y recogida de datos

En total se recogieron 25 muestras de tejido en fresco de piel normal de 25 voluntarios mayores de edad que requerían cirugía cutánea menor. Estos voluntarios se seleccionaron de forma consecutiva, es decir, se fue escogiendo a cada individuo que cumplía con los criterios de selección del estudio hasta que se alcanzó el número necesario. De dichas muestras, 12 fueron de una zona corporal expuesta a la radiación solar de forma crónica (cara, cuello y dorso de manos), otras 11 se recogieron de una zona intermitentemente expuesta al sol (tronco, parte superior de las extremidades, hombros) y otras 2 muestras provinieron de zonas no expuestas al sol (glúteos y axilas). Las muestras de tejido se obtuvieron de las partes sobrantes de resecciones de piel realizadas para eliminar por escisión una lesión cutánea. Estas muestras se recogieron en pacientes del Hospital General de Castellón y del Hospital La Plana de Vila-real, por parte de los especialistas en dermatología que participaron en el proyecto.

Todos los participantes del estudio dieron su consentimiento por escrito para ser incluidos en la investigación. Este proyecto fue aprobado por los comités éticos de investigación clínica (CEIC) de ambos hospitales involucrados (Hospital General de Castellón y Hospital de La Plana de Vila-real) y por el Comité Deontológico de la Universitat Jaume I.

Inmediatamente después de resecar el tejido, todas las biopsias cutáneas se sumergieron en la solución RNAlater para estabilizar el material biológico (Thermo Fisher Scientific, Walham, MA, EE. UU.). El transporte de las muestras al laboratorio del Departamento de Medicina se llevó a cabo en neveras portátiles por un miembro del grupo de investigación. Una vez las muestras llegaron al laboratorio, los tejidos se cortaron para producir secciones de aproximadamente $5 \mathrm{~mm}^{2}$ de área. La disección del tejido se realizó con un bisturí estéril. Inmediatamente después, las piezas de tejido fueron sumergidas en tiocinato de amonio al 3,8 \% (Sigma-Aldrich, St Louis, MO, EE. UU.) en solución salina tamponada con fosfato $(\mathrm{pH} 7,4)$ y se incubaron durante 3 horas a temperatura ambiente para poder separar la epidermis de la dermis. Las extracciones de ADN de las epidermis se realizaron utilizando el QIAamp DNA Mini Kit (Qiagen, Hilden, Alemania), siguiendo las recomendaciones del fabricante.

\section{Análisis de mutaciones somáticas mediante secuenciación masiva}

Para identificar las mutaciones somáticas en las biopsias de piel, se realizó una secuenciación dirigida de los exones de un panel de 47 genes: ADAM29, ADAMTS18, ARID1A, ARID2, BAI3/ADGRB3, BRAF, CDKN2A, CRNKL1, ERBB4, EPHA2, EZH2, FAT1, FAT2, FGFR3, GRIN2A, GRM3, HRAS, IL7R, KRAS, MECOM, MLL2/KMT2B, NEBL, NF1, NOTCH1, NOTCH2, NOTCH3, NRAS, PIK3CA, PLCB1, PPP6C, PPP1R3A, PREX2, PTCH1, PTEN, PTPRB, PTPRK, RAC1, RB1, RBM10, SALL1, SCN1A, SF3B1, SPHKAP, STAT5B, TP53, TERT, ZNF750. Estos genes fueron seleccionados en base a los siguientes criterios: 1) genes involucrados en diferentes tumoraciones y tipos de cáncer de piel (nevus, melanoma, carcinoma basocelular, carcinoma espinocelular) (Jayaraman, Rayhan, Hazany y Kolodney 2014; Lin et al. 2014); 2) genes que están involucrados en la progresión tumoral de una amplia gama 
de tipos de cáncer (Davies et al. 2002), o 3) genes mutados con frecuencia en muestras de piel normal (Martincorena et al. 2015).

La secuenciación de los genes diana se realizó utilizando la tecnología de secuenciación de nueva generación (NGS, por sus siglas en inglés), con el objetivo de detectar mutaciones en diversos genes al mismo tiempo. La técnica consistió en el aislamiento, el enriquecimiento y la secuenciación de regiones específicas del genoma en una muestra. El proceso de secuenciación se llevó a cabo en el Centro de Regulación Genómica de Barcelona (CRG-CNAG). Las secuencias obtenidas para cada muestra se compararon con la secuencia consenso de cada uno de los genes diana analizados disponibles en la base de datos GenBank (http://www.ncbi.nlm.nih.gov/genbank/). Se seleccionaron aquellas alteraciones presentes en más del $1 \%$ de todas las lecturas realizadas. Los análisis bioinformáticos se llevaron a cabo en el CRG-CNAG por el Departamento de Análisis de Datos.

\section{Análisis del genotipo MC1R}

El ensayo genotípico del gen $M C 1 R$ se realizó mediante la secuenciación completa de la región codificante del gen, ya que se trata de un gen altamente polimórfico. Una vez amplificada la región codificante del gen, los productos de la PCR fueron purificados utilizando exonucleasa I y fosfatasa alcalina (Roche Molecular Bioquímicos AQ2, Mannheim, Alemania). El análisis de secuenciación se realizó utilizando la tecnología Sanger Sequencing. Como medida de control de calidad se incluyó una muestra con genotipo conocido (control positivo) y un pocillo sin muestra (control negativo) por placa de 96 pocillos. Los individuos fueron clasificados según eran portadores o no de variantes $\mathrm{R}$ del gen $M C 1 R$.

\section{Análisis estadístico}

El test de Shapiro-Wilk fue utilizado para determinar si la variable principal del estudio seguía una distribución normal en nuestra población. La prueba $U$ de Mann-Whitney se utilizó para comparar la tasa mutacional entre dos grupos de muestras independientes. La prueba de Kruskal-Wallis se utilizó para comparar la tasa mutacional entre más de dos muestras independientes. Los análisis estadísticos se realizaron con el software SPSS v25. Resultados con un p-valor menor de 0,05 fueron considerados como estadísticamente significativos. Los individuos con algún dato no conocido fueron eliminados del correspondiente análisis.

\section{Resultados}

Este proyecto piloto se centra en analizar la tasa de mutaciones somáticas acumuladas en 25 muestras de tejido cutáneo normal según la fotoexposición de la muestra en base a la zona corporal de la cual procede el tejido resecado y según el genotipo del gen $M C 1 R$.

En primer lugar, se analiza si la tasa mutacional (número total de mutaciones somáticas) por muestra sigue una distribución normal en nuestra población, para determinar qué tipo de pruebas estadísticas deben llevarse a cabo para realizar la comparación entre subconjuntos de datos. Según el test de normalidad Shapiro-Wilk, la tasa de mutaciones somáticas no sigue una distribución normal en nuestra población $(P=0,014)$. Por este motivo, para realizar los análisis estadísticos, se aplicaron test estadísticos no paramétricos. 
Después, se analizó la tasa mutacional según el patrón de exposición solar del tejido resecado (exposición solar crónica, intermitente o sin fotoexposición) mediante una prueba de Kruskal-Wallis (figura 1). Los resultados muestran que existen diferencias en el número total de mutaciones somáticas acumuladas según el grado de exposición solar del tejido resecado $(P=0,012)$. En general, se acumulan significativamente más mutaciones somáticas en zonas con mayor grado de exposición solar, confirmando el papel de la radiación UV en la aparición de mutaciones somáticas en las células de la epidermis. Así pues, en aquellas muestras de regiones crónicamente fotoexpuestas, hay una mayor acumulación de mutaciones somáticas en comparación con las muestras intermitentemente fotoexpuestas $(P=0,013)$ y con las muestras no expuestas al sol $(P=0,044)$.

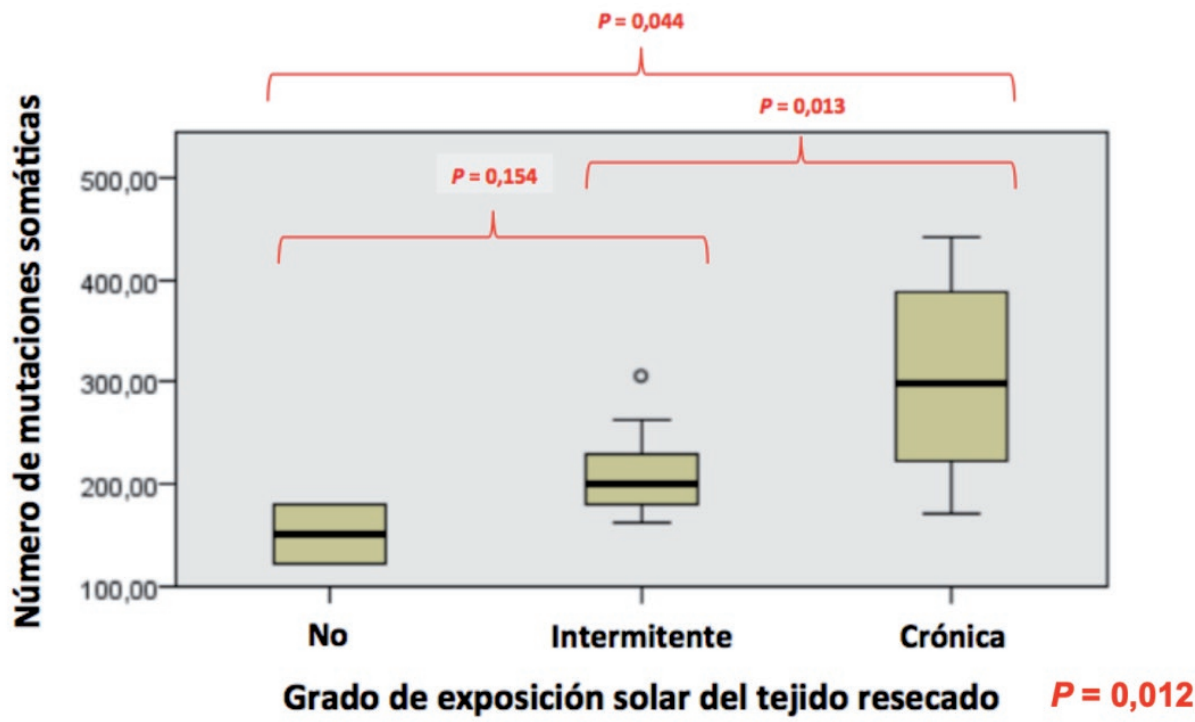

Figura 1. Diagrama de cajas en el que se representa el número total de mutaciones según la procedencia de la muestra de piel obtenida: región no fotoexpuesta, región intermitentemente fotoexpuesta o región crónicamente fotoexpuesta.

Por otro lado, debido a la elevada asociación del genotipo $M C 1 R$ con la sensibilidad cutánea a la exposición solar, se analizó la relación entre el número total de mutaciones somáticas y el genotipo del gen $M C 1 R$, dividiendo a los individuos en dos grupos según eran portadores o no de variantes $\mathrm{R}$ del gen $M C 1 R$. 


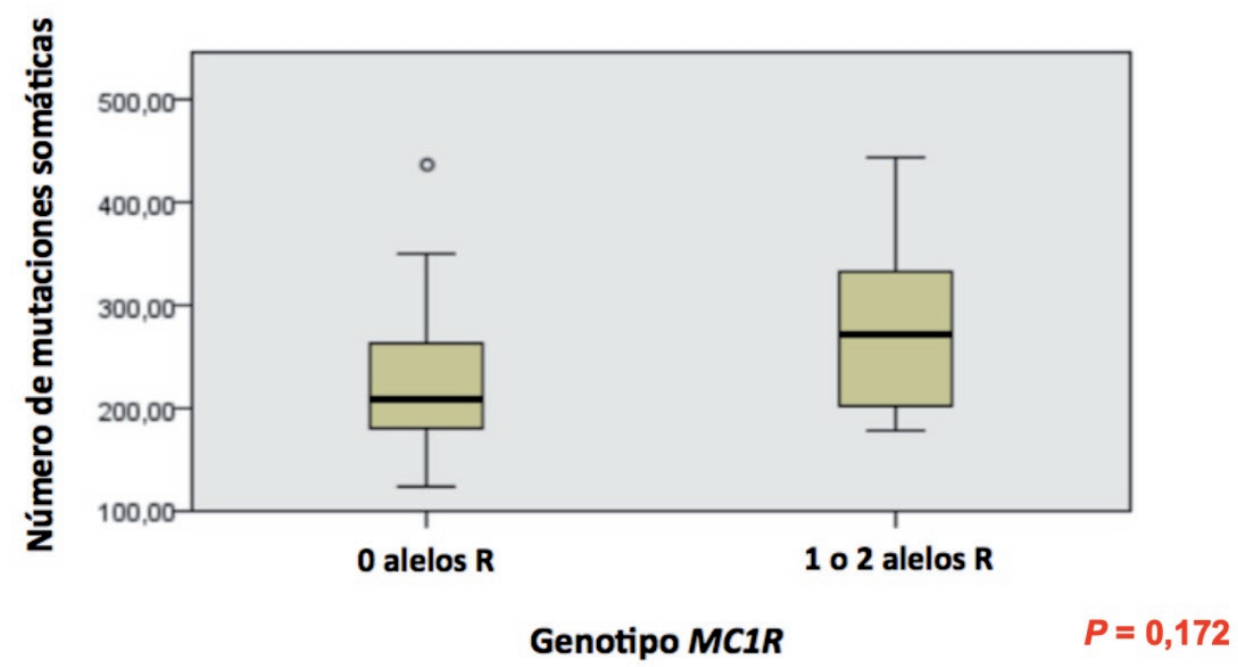

Figura 2. Diagrama de cajas en el que se representa el número de mutaciones somáticas acumuladas en los individuos que no portaban ninguna variante $\mathrm{R}$ en el gen $M C 1 R$ y aquellos individuos portadores de al menos 1 variante $R$.

Tal y como se observa en la figura 2, no existe una diferencia estadísticamente significativa en el número de mutaciones acumuladas en las muestras de piel normal según el genotipo del gen $M C 1 R$ de los individuos $(P=0,172)$. Sí que es cierto que se observa una tendencia positiva entre el número de mutaciones somáticas y el ser portador de una o dos variantes $\mathrm{R}$, aunque dicha observación tiene que ser confirmada incluyendo más voluntarios en el proyecto de investigación.

\section{Discusión y conclusiones}

Debido a la importancia de la acumulación de mutaciones somáticas en la aparición y el desarrollo del cáncer de piel, sumado a los pocos estudios realizados en estados preneoplásicos, este estudio piloto se centra en analizar el papel que juega la exposición a la radiación solar y la sensibilidad solar del individuo en la acumulación de mutaciones somáticas en piel normal con el objetivo de revelar ideas fundamentales en etapas más tempranas del desarrollo del cáncer de piel.

En primer lugar, este estudio piloto indica que existen diferencias significativas en el número total de mutaciones somáticas acumuladas según el grado de exposición solar del tejido cutáneo resecado. Así pues, se confirma el papel clave que tiene la radiación UV en la aparición de mutaciones somáticas, ya que se observa una mayor acumulación de mutaciones somáticas en aquellas zonas con mayor grado de exposición solar. Concretamente, el número de mutaciones somáticas acumuladas ha resultado ser significativamente mayor en biopsias cutáneas procedentes de zonas corporales crónicamente fotoexpuestas que en muestras de zonas fotoexpuestas de forma intermitente, así como muestras de regiones no expuestas al sol. Sin embargo, no se obtuvieron diferencias significativas al comparar la tasa mutacional entre muestras de zonas intermitentemente fotoexpuestas y no fotoexpuestas, a pesar de seguir la tendencia general observada (véase la figura 1). El hecho de no haber alcanzado un resultado significativo para esta última asociación tal vez se deba al bajo número de muestras del que se dispone en este estudio piloto, ya que para el grupo de piel no fotoexpuesta tan solo se cuenta con 2 biopsias. 
Destacar que nuestros resultados se apoyan en estudios previos que analizan la tasa mutacional en cáncer de piel. Por ejemplo, Hayward y colaboradores (2017) afirman que existe una diferencia significativa en el número de mutaciones somáticas encontradas en melanoma dependiendo de la región corporal donde se desarrolla, siendo mayor las mutaciones acumuladas en melanomas de regiones crónicamente fotoexpuestas que en melanomas de regiones no expuestas al sol de forma crónica. En el caso de nuestra investigación, se ha podido demostrar esta relación entre el patrón de exposición solar y la tasa mutacional en piel normal (tejido cutáneo sano), hecho destacable por su novedad (se dispone todavía de muy poca evidencia científica) y su interés en el campo de la dermatología oncológica (se abre la puerta para conocer en profundidad las etapas más tempranas del desarrollo del cáncer cutáneo).

Por otro lado, se analizaron las diferencias en la acumulación de mutaciones somáticas según las variantes genéticas del gen $M C 1 R$ que portaba cada individuo. Como se ha comentado en la introducción, las variantes $\mathrm{R}$ de $M C 1 R$ están relacionadas con tener una mayor sensibilidad a la exposición solar y una defectuosa reparación de daños en el ADN (García-Borrón, Abdel-Malek y Jiménez-Cervantes 2014; Robles-Espinoza et al. 2016; Tagliabue et al. 2015). Según nuestros resultados, no se observan diferencias significativas en el número de mutaciones somáticas entre los individuos portadores y los no portadores de alguna variante $\mathrm{R}$ en el gen MC1R. No obstante, sí se observa que los sujetos con alguna variante $R$ tienden a acumular un mayor número de mutaciones que los individuos que no son portadores de dicha variante. Esta tendencia observada concuerda con estudios previos que indican que los melanomas de sujetos con al menos una variante $R$ en el gen $M C 1 R$ acumulan una mayor cantidad de mutaciones somáticas que aquellos melanomas de individuos no portadores de variantes R (Robles-Espinoza et al. 2016).

Una de las principales limitaciones del estudio es el bajo tamaño de muestra de este estudio piloto, aunque debe tenerse en cuenta que actualmente se están analizado un total de 125 biopsias cutáneas más con las que esperamos confirmar los resultados obtenidos hasta el momento.

Como fortalezas del estudio cabe destacar que se ha procurado no caer en ciertos sesgos de selección como el del voluntario o el sesgo de Berkson, puesto que a los individuos los ha escogido el propio especialista y el tejido sano estudiado siempre procedía de una resección realizada por una lesión que no tuviera relación con el cáncer de piel.

Como futuras líneas de investigación, consideramos que sería muy interesante determinar si existen mutaciones específicas, lo que se conoce con el nombre de hotspots o puntos calientes, es decir, regiones del genoma que muestran una mayor tendencia a padecer una mutación somática. Frente a este tipo de mutaciones recurrentes, tal vez fuera posible desarrollar fármacos o terapias dirigidas, lo que supondría un gran avance en el tratamiento del cáncer de piel.

\section{Referencias bibliográficas}

Davies, Helen, Graham R. Bignell, Charles Cox, Philip Stephens, Sarah Edkins, Sheila Clegg, Jon Teague, Hayley Woffendin, Matthew J. Garnett, William Bottomley, Neil Davis... y P. Andrew Futreal. 2002. "Mutations of the BRAF gene in human cancer». Nature 417(6892): 949-954. http://doi.org/10.1038/nature00766 
García-Borrón, José Carlos, Zalfa A. Abdel-Malek y Celia Jiménez-Cervantes. 2014. «MC1R, the CAMP pathway and the response to solar UV: Extending the horizon beyond pigmentation». Pigment cell \& melanoma research 27(5): 699720. http://doi.org/10.1111/pcmr.12257

González-Púmariega, Maribel, Marioly Vernhes Tamayo y Ángel Sánchez-Lamar. 2009. "La radiación ultravioleta. Su efecto dañino y consecuencias para la salud humana». Theoria 18(2): 69-80.

Hayward, Nicholas K., James S. Wilmott, Nicola Waddell, Peter A. Johansson, Matthiew A. Field, Katia Nones, Ann-Marie Patch... y Graham J. Mann. 2017. «Whole-genome landscapes of major melanoma subtypes». Nature 545(7653): 175-180. http://doi.org/10.1038/nature22071

Hernando Fuster, Bárbara. 2017. "The genetic basis of sunlight sensitivity and melanoma-risk pigmentation phenotypes: The role of sex-specific genetic effects, 3' untranslated regions and melanoma susceptibility genes». Tesis doctoral. Castellón: Universitat Jaume I.

Jayaraman, Shyam Sundar, David J. Rayhan, Salar Hazany y Michael S. Kolodney. 2014. «Mutational Landscape of Basal Cell Carcinomas by Whole-Exome Sequencing». Journal of Investigative Dermatology 134(1): 213-220. http://doi.org/10.1038/jid.2013.276

Karki, Roshan, Deep Pandya, Robert C. Elston y Cristiano Ferlini. 2015. «Defining "mutation" and "polymorphism" in the era of personal genomics". BMC Medical Genomics 8. http://doi.org/10.1186/s12920-015-0115-z

Lin, De-Chen, Jia-Jie Hao, Yasunobu Nagata, Liang Xu, Li Shang, Xuang Meng, Yusuke Sato, Yusuke Okuno... y H. Phillip Koeffler. 2014. "Genomic and molecular characterization of esophageal squamous cell carcinoma». Nature genetics 46(5): 467-473. http://doi.org/10.1038/ng.2935

Madan, Vishal, John T. Lear y Rolf-Markus Szeimies. 2010. «Non-melanoma skin cancer». The Lancet 375(9715): 673-685. http://doi.org/10.1016/S01406736(09)61196-X

Martincorena, Íñigo, Amit Roshan, Moritz Gerstung, Peter Ellis, Peter Van Loo, Stuart McLaren, David C. Wedge, Anthony Fullam... y Peter J. Campbell. 2015. «High burden and pervasive positive selection of somatic mutations in normal human skin». Science 348(6237): 880-886. http://doi.org/10.1126/science.aaa6806

Robles-Espinoza, Carla Daniela, Nicola D. Roberts, Shuyang Chen, Finbarr P. Leacy, Ludmil B. Alexandrov, Natapol Pornputtapong, Ruth Halaban, Michael Krauthammer, Rutao Cui, David Timothy Bishop y David J. Adams. 2016. "Germline MC1R status influences somatic mutation burden in melanoma». Nature Communications 7. http://doi.org/10.1038/ncomms 12064

Scherer, Dominique y Rajiv Kumar. 2010. "Genetics of pigmentation in skin cancer--a review». Mutation $\quad$ Research 141-153. http://doi.org/10.1016/j.mrrev.2010.06.002

Stratton, Michael R., Peter J. Campbell y P. Andrew Futreal. 2009. «The cancer genome». Nature 458(7239): 719-724. http://doi.org/10.1038/nature07943

Tagliabue, Elena, Maria Concetta Fargnoli, Sara Gandini, Patrick Maisonneuve, F. Liu, M. Kayser, Tamar Nijsten... y Sara Raimondi. 2015. «MC1R gene variants and non-melanoma skin cancer: a pooled-analysis from the M-SKIP project». British Journal of Cancer 113(2): 354-363. http://doi.org/10.1038/bjc.2015.231 